Int. J. Dev. Biol. 54: 897-904 (2010)

doi: $10.1387 / \mathrm{ijdb} .082839 \mathrm{kp}$

\title{
Evolving expression patterns of the homeotic gene Scr in insects
}

\author{
KARLA D. PASSALACQUA\#, STEVEN HRYCAJ\#, NAJMUS MAHFOOZ and ALEKSANDAR POPADIC* \\ Department of Biological Sciences, Wayne State University, Detroit, USA
}

\begin{abstract}
While the mRNA expression patterns of homeotic genes have been examined in numerous arthropod species, data on their protein accumulation is extremely limited. To address this gap, we analyzed the protein expression pattern of the hox gene Sex combs reduced (Scr) in six hemimetabolous insects from four divergent orders (Thysanura, Orthoptera, Dictyoptera and Hemiptera). Our comparative analysis reveals that the original domain of SCR expression was likely confined to the head and then subsequently moved into the prothorax (T1) in winged insect lineages. The data also show a trend toward the posteriorization of the anterior boundary of SCR expression in the head, which starts in the mandibles (Thysanura) and then gradually shifts to the maxillary (Orthoptera) and labial segments (Dictyoptera and Hemiptera), respectively. In Thermobia (firebrat) and Oncopeltus (milkweed bug) we also identify instances where SCR protein is not detected in regions where mRNA is expressed. This finding suggests the presence of a posttranscriptional regulatory mechanism of Scr in these species. Finally, we show that SCR expression in insect T1 legs is highly variable and exhibits divergent patterning even among related species. In addition, signal in the prothoracic legs of more basal insect lineages cannot be associated with any T1 specific features, indicating that the acquisition of SCR in this region preceded any apparent gain of function. Overall, our results show that Screxpression has diverged considerably among hemimetabolous lineages and establish a framework for subsequent analyses to determine its role in the evolution of the insect head and prothorax.
\end{abstract}

KEY WORDS: Sex combs reduced (Scr), gene expression, hemimetabolous insects, evolution, prothorax (T1)

\section{Introduction}

Numerous studies have shown that changes in the expression and function of homeotic (hox) genes were pivotal in the evolution of the insect bauplan (Beeman et al., 1993; Beeman et al., 1989; Carroll et al., 2001; Hughes and Kaufman, 2000; Hughes and Kaufman, 2002; Mahfooz et al., 2007; Mahfooz et al., 2004; Rogers et al., 1997; Rogers et al., 2002; Struhl, 1982; Tomoyasu et al., 2005). This insight was primarily based on mRNA expression patterns, however, data on protein accumulation are much more limited. In fact, only two hox genes ( $U b x$ and $a b d-A)$ have been studied at such a broad level, largely due to the availability of the cross-reacting FP6.87 antibody (Kelsh et al., 1994). The results obtained identified key changes in expression patterns of Ubx that directly correlated with changes in arthropod body plans (Abzhanov et al., 1999; Angelini and Kaufman, 2005; Averof and
Patel, 1997; Castelli-Gair and Akam, 1995; Damen et al., 1998; Mahfooz et al., 2004; Peterson et al., 1999; Stern, 1998; Telford and Thomas, 1998; Zheng et al., 1999). In insects, Ubx expression has also been linked to the differential enlargement of insect hind (T3) legs, which were subsequently confirmed by functional studies (Mahfooz et al., 2007; Mahfooz et al., 2004). As illustrated by these findings, the availability of cross-reacting antibodies are critical in that they provide better phylogenetic sampling by circumventing the laborious process of cloning and characterizing orthologous genes. Such broader sampling can provide a deeper understanding as to how changes of expression patterns can effect evolving morphological structures across a wider range of

\footnotetext{
Abbreviations used in this paper: dT1, dorsal prothorax; Lb, labium; Mn, mandibles; Mx, maxillae; Scr, sex combs reduced; T1, prothorax; T2, mesothorax; T3, metathorax.
}

\footnotetext{
*Address correspondence to: Aleksandar Popadic. Department of Biological Sciences, Wayne State University, Detroit, MI 48202, USA. Fax: +1-313-577-6891. e-mail: apopadic@biology.biosci.wayne.edu \#Note: Both authors contributed equally to this paper.
}

Supplementary Material for this paper (one figure) is available at: http://dx.doi.org/10.1387/ijdb.092839kc

Accepted: 29 April 2009. Final author-corrected PDF published online: 9 March 2010.

ISSN: Online 1696-3547, Print 0214-6282

(C) 2010 UBC Press

Printed in Spain 
taxa.

To address this gap, we focused on the homeotic (hox) gene Sex combs reduced (Scr). In insects, Scrfunctions in two distinct body regions, the head and the thorax. Previous insights from mRNA expression and functional studies have indicated that this gene is crucial in establishing the identity of the labial segment, suppressing wing formation on the prothorax (T1), and directing the development of T1 leg combs (Beeman et al., 1993; Beeman et al., 1989; Curtis et al., 2001; Hughes and Kaufman, 2000; Pattatucci and Kaufman, 1991; Pattatucci et al., 1991; Rogers et al., 1997; Rogers et al., 2002; Shippy et al., 2006). The data on protein expression in two model systems (Drosophila and Tribolium) show that, in general, mRNA patterns closely coincide with the protein pattern (Curtis etal., 2001; Mahaffey and Kaufman, 1987). However, a report in the terrestrial isopod $P$. scaberhas described a difference between mRNA and protein expression patterns of $S c r$, revealing the existence of post-transcriptional regulation in this species (Abzhanov and Kaufman, 1999). This result highlights the importance of complementing previously published ScrmRNA expression patterns with data on the accumulation of SCR protein. In the present study, we utilize a previously described cross-reacting antibody (Abzhanov and Kaufman, 1999; Curtis et al., 2001) to provide data on the protein accumulation of SCR in six hemimetabolous insect species. Our broad sampling encompasses four divergent insect orders (listed from early to late-branching): Thysanura, the firebrat Thermobia domestica; Orthoptera, the cricket Acheta domestica and the grasshopper Schistocerca americana; Dictyoptera, the cockroach Periplaneta americana and the praying mantis Tenodera aridifolia; and finally Hemiptera, the milkweed bug Oncopeltus fasciatus. Our analysis has revealed the following key aspects of the evolution of SCR patterning in insects: (i) the anterior border of SCR expression starts in the mandibular segment (Thermobia) and moves posterior to the maxillary segment in some lineages (Achetaand Schistocerca) and then shifts even more posterior to the labial segment (Periplaneta, Tenodera and Oncopeltus); (ii) we found two instances (Thermobia, Oncopeltus) in which Scr mRNA is clearly expressed in a defined region (Rogers et al., 1997) but never accumulates protein, similar to the post-transcriptional regulatory situation reported in crustaceans (Abzhanov and Kaufman, 1999); (iii) SCR protein expression in T1 legs is very dynamic and can only occasionally be linked to particular morphological structures. Overall, our data suggests that SCR protein accumulation is highly labile and can be gained or lost even in closely related species.

\section{Results}

\section{Scr expression in Thermobia domestica (firebrat), a basal insect lineage}

The thysanuran Thermobia domestica (firebrat) is a primitively wingless species that represents a basal insect lineage. Data on SCR patterning in this species can therefore provide insight into the ancestral expression pattern of this gene. SCR protein accumulation in the firebrat is very dynamic and can be detected from $\sim 30 \%$ - 75\% stages of development. At $\sim 30 \%$, when limb buds are just beginning to elongate, the anterior border of SCR expression is in a small cluster of cells in the posterior, mid-ventral portion of the mandibular segment (Fig. 1A). Signal is also detected in a narrow mid-ventral region in the maxillary segment and throughout the entire developing labial segment and its appendages (Fig. 1A). At $\sim 35 \%$ development SCR accumulation in the mandibles slightly diminishes, whereas expression in the maxillary segment becomes stronger and expands laterally into the lobes of the developing appendages (Fig. 1B). Expression remains strong throughout the entire labial segment at this stage. At $\sim 40 \%$ development, SCR is expressed throughout the labium while it is entirely lost in the mandibular segment (Fig. 1C). The signal is also detected in the proximal lobes of the maxillary appendages and in the posterior portion of the corresponding mid-ventral region at this stage. At $\sim 45 \%$ development, signal is reduced in the posterior maxillary segment and is still predominantly confined to the growing labial appendages (Fig. 1D). In late developmental stages ( 75\%) SCR reappears in the anterior head, with strong expression in the mandibular appendages and a weaker signal in the proximal lobes of the maxillary segment (Fig. 1E). At this stage there is no longer any SCR expression in the labium. It important to note that SCR protein never accumulates in the dorsal T1 region or in the T1 leg at any time during development (Fig. 1F).

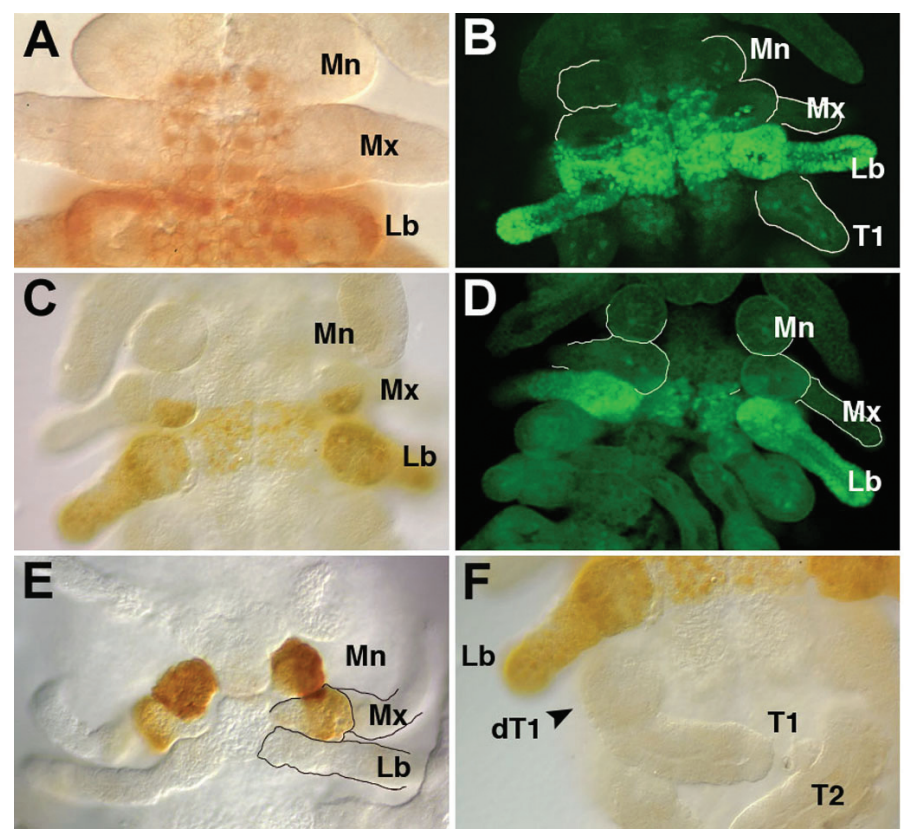

Fig. 1. SCR protein expression in Thermobia domestica (firebrat). (A) At $\sim 30 \%$, SCR is detected in a small patch of cells in the posterior/ventral region of the mandibular segment, in the mid-ventral portion of the maxillary segment and throughout the entire labium. (B) Later in development ( 35\%), signal in the mandibles slightly diminishes, while in the maxillary segment it expands laterally into the base of the appendages. Expression remains throughout the labium at this stage. (C) At $~ 40 \%$ development, SCR is entirely lost in the mandibles, has become restricted to the posterior half of the maxillae and remains throughout the labium. (D) At $\sim 45 \%$, expression is primarily restricted to the labium, with only a slight signal in the posterior maxillae. (E) During late stages of development ( 75\%), SCR reappears in the anterior head and is strongly detected in the mandibles with a more moderate signal in the proximal lobes of the maxillary appendages. SCR is completely absent in the labium at this stage. (F) SCR protein never accumulates along the first thoracic leg or in the dorsal T1 region during development. Abbreviations: $M n$, mandible; $M x$, maxillae; Lb, labium; T1, first thoracic leg; dT1, dorsal T1 region. 


\section{Expression of SCR in the insect head shows a trend of posteriorization}

As shown in Fig. 2, SCR is predominantly expressed in the labial segment of all insect species examined in this report. However, the initial anterior border of head expression moves in a posterior direction in a species-specific manner. After Thermobia, the next two insects analyzed were orthopterans, the cricket Acheta domestica and the grasshopper Schistocerca americana. At early stages of development, cricket SCR expression is restricted to the posterior region of the maxillary segment and to the proximal lobes of its appendages (Fig. 2A). However, the signal in the maxillae of the grasshopper is much more diffuse in the midventral region (Fig. 2C). In addition, SCR extends more distally in the maxillary appendages encompassing a broader expression domain compared to crickets. In both insects, SCR is still predominantly expressed throughout the labial appendage at this stage. Later in development, expression is entirely lost in the maxillary appendages and only slightly remains in the mid ventral region of this segment (Fig. 2B arrowhead, Fig. 2D). However, SCR continues to be strongly expressed throughout the labial segment and its developing appendages in both insects (Fig. 2 $B, D)$.

The next insect lineage analyzed was Dictyoptera, represented by the cockroach Periplaneta americana and the praying mantis Tenodera aridifolia. At early stages of development, SCR is expressed throughout the entire labial segment but is absent in the maxillary segment in both species (Fig. $2 \mathrm{E}, \mathrm{G}$ ). This result

Fig. 2. SCR protein expression patterns in the head segments of five hemimetabolous species. (A,B) Acheta domestica (cricket), (C,D) Schistocerca americana (grasshopper), (E,F) Periplaneta americana (cockroach), (G,H) Tenodera aridifolia (praying mantis) and (I,J) Oncopeltus fasciatus (milkweed bug). Left hand column (A,C,E,G,I) represents early development (25-30\%). Right hand column (B, D,F,H,J) represents midlate development (35-45\%). (A) Early Acheta embryo showing the localization of SCR to the posterior half of the maxillary segment and the proximal region of its associated appendage. Strong expression is observed throughout the entire labial segment and its appendages. (B) At mid-development, SCR is no longer detected in the maxillary appendages and is confined to a small cluster of cells in the mid-ventral portion of this segment (arrow). SCR remains strongly expressed throughout the labium and its appendages. (C) In early Schistocerca embryos, SCR signal is observed in the proximal region of the maxillary and throughout the labial appendages. Ventral SCR patterning in both the maxillary and labial segments appears faint at this stage (D) Mid-staged Schistocerca embryo showing strong signal throughout the labium and its associated appendages. SCR is restricted to the posterior most region of the midventral maxillary segment. (E) Early Periplaneta embryo showing strong SCR expression throughout the labial appendages and low levels of signal in the mid-ventral region of this segment. (F) Later in development, signal is restricted to the distal portions of the labial appendages, with no mid-ventral expression (arrowhead). (G) In early Tenodera embryos, SCR is strongly expressed throughout the labial segment and its associated appendages. (H) While strong signal persists in the ventral labium, SCR expression in the appendages is restricted to the proximal lobes. (I) Early Oncopeltus embryo showing strong SCR expression throughout the labial appendages with moderate signal in the mid-ventral region of this segment. Additional SCR signal can be detected at the lateral edge between the maxillary and labial segments (arrowhead). (J) At middevelopment, SCR is completely lost in the mid-ventral region (arrowhead) of the labial segment but remains strongly expressed throughout its appendages. Abbreviations: Mn, mandible; $M x$, maxillae; Lb, labium. reveals a further posteriorization of the anterior border of SCR expression when compared to more basal insect lineages (Thysanura, Orthoptera). In addition, the mid-ventral region of the labial segment in the cockroach exhibits reduced SCR expression, while the mantis maintains strong signal in this area (Fig.
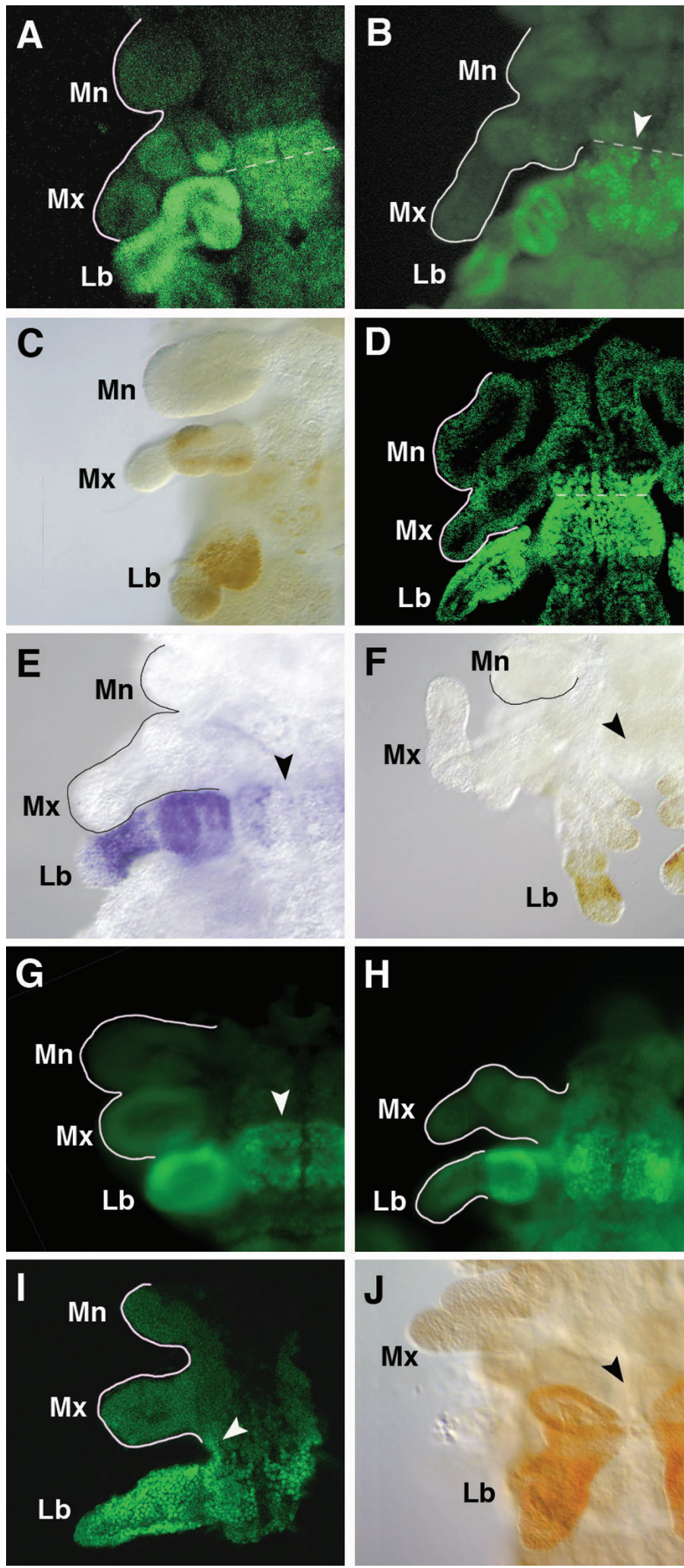
Fig. 3. SCR protein expression in the dorsal T1 (dT1) region and prothoracic (T1) leg in hemimetabolous species. (A) An arrowhead points to SCR expression in the dorsal T1 (dT1) region of Acheta domestica (cricket), Schistocerca americana (grasshopper), Periplaneta americana (cockroach) andTenodera aridifolia (praying mantis). In'Schistocerca, while SCR is only detected in discrete regions of $d T 1$, strong expression is seen throughout this region in all other insect lineages examined in this study. (B) Early Acheta embryo ( 35\% development) showing SCR expression in the anterior region of the T1 leg that corresponds to the future tibial-tarsal joint (arrowhead). (C) Schistocerca T1 leg showing a complete lack of SCR expression. (D) SCR protein expression at early and mid-developmental stages in the T1 legs of Periplaneta. (D, left) At early stages no SCR protein is expressed in the T1 legs of Periplaneta. (D, right) Later in development, strong SCR signal is now detected in the proximal coxa, distal femur and throughout most of the tibia (arrowheads). (E) SCR protein expression at early and middevelopmental stages in the T1 legs of Tenodera. (E, upper left) At early stages ( 30\%) SCR signal is restricted to a small patch of cells in the anterior tibia (arrowhead). (E, lower left) SCR expression expands and accumulates in the posterior ridge of the coxa, femur and tarsus at later stages. (E, right) Dissected T1 leg of Tenodera at the first nymphal stage. Solid lines link embryonic SCR expression patterns at mid-developmental stages to the corresponding regions of first nymph T1 legs. (F) SCR protein expression in the dT1 region and T1 leg of Oncopeltus. (F, lower left) SCR protein accumulates in the dT1 region of Oncopeltus (arrowhead) and in the distal tibia of the
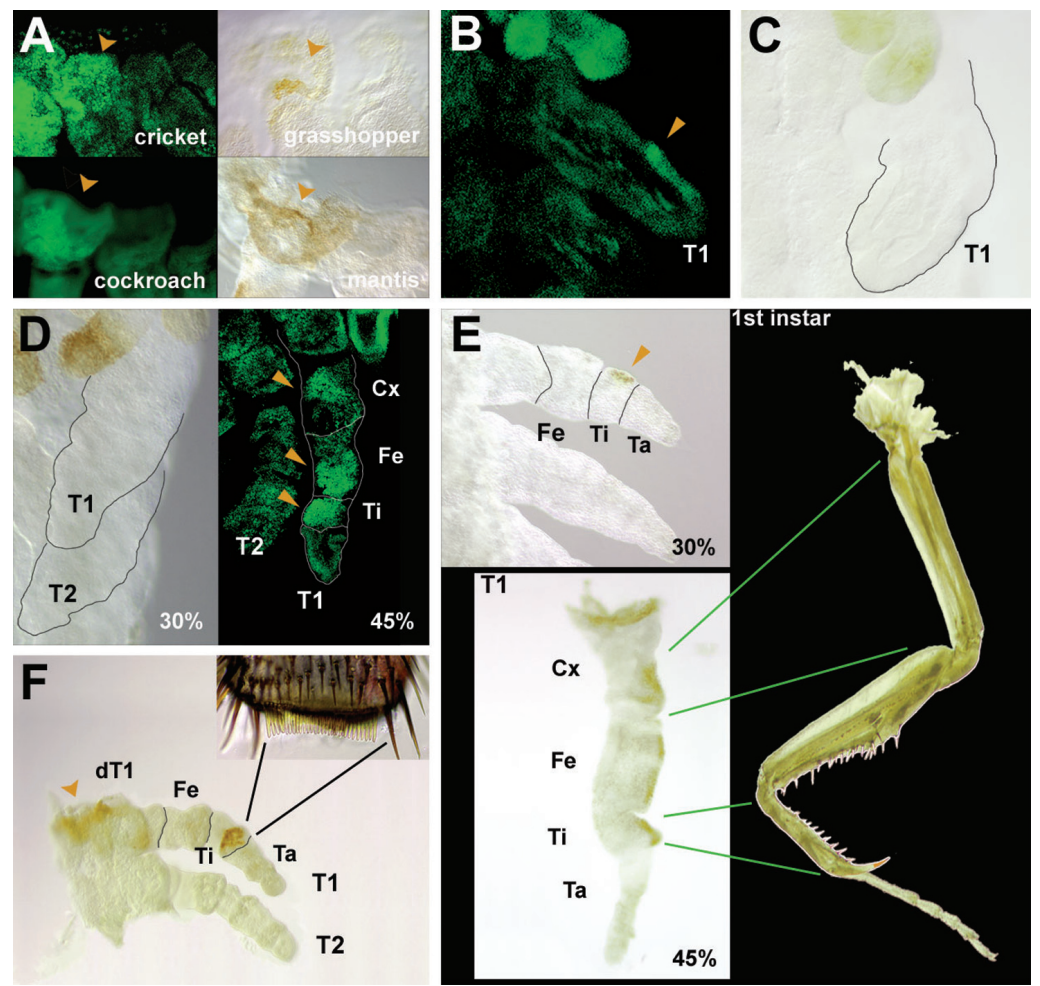

T1 leg. (F, upper right) Close up of the sex combs that appear on the distal tibia of the T1 legs of Oncopeltus first nymphs. Solid lines link embryonic SCR expression in the distal tibia to the corresponding structure (sex combs) on the prothoracic legs of first nymphs. Abbreviations: Cx, coxa; Fe, femur; Ti, tibia; Ta, tarsus; T1, first thoracic leg; T2, second thoracic leg; dT1, dorsal T1 region.

$2 \mathrm{E}, \mathrm{G}$, arrowheads). Later in development, cockroach SCR signal is completely absent from the mid ventral region (Fig. $2 F$, arrowhead) and is confined to the distal portion of the developing labial appendages only. In contrast, mantis embryos maintain strong mid-ventral expression in the labial segment. The signal in the elongating labial appendage is restricted to the proximal lobe (Fig. $2 \mathrm{H}$ ), displaying a different pattern from the cockroach.

The hemipteran milkweed bug (Oncopeltus fasciatus) represents a more derived hemimetabolous lineage. In early development, SCR protein is primarily localized in the labial segment, although some faint expression can be detected at the lateral edge between the maxillary and labial segments (Fig. 2l, arrowhead). Additionally, moderate SCR signal is present in the mid ventral portion of the labial segment at this stage. This early restriction of SCR to the labium differs from previously reported mRNA expression patterns that show clear signal in the posterior region of the maxillary appendage (Rogers et al., 1997). This situation is reminiscent of the previously described differences of expression in the dT1 region of Thermobia, and is likely due to a post-transcriptional mechanism. At later developmental stages ( $(35-45 \%)$, SCR protein is strictly confined to the growing labial appendages with a complete absence of signal in the mid ventral region of the labial segment (Fig. 2J, arrowhead).

\section{The prothorax (T1): expression in the dorsal (dT1) region is highly conserved}

Functional analyses in Drosophilaand Triboliumhave indicated that one of the primary functions of Scris to suppress the formation of wings on the adult prothoracic (T1) segment (Beeman et al.,
1989; Carroll et al., 1995; Rogers et al., 1997; Struhl, 1982; Tomoyasu et al., 2005). It is generally accepted that this is a conserved role due to the fact that the wingless $\mathrm{T} 1$ segment is a key insect feature. Hence, it is important to understand the origin(s) of SCR expression in this region as it relates to the evolution of the insect body plan. Thermobia (firebrats) represent an ideal starting point to assess the origins of the wing repressive function of SCR as these insects are considered to be primitively wingless. A previous analysis of ScrmRNA expression patterns in Thermobia showed clear signal in the dorsal T1 (dT1) region (Rogers et al., 1997). However, as depicted in Fig. 1F, SCR protein does not accumulate in this region at any time during embryogenesis. In one orthopteran species Acheta (crickets), SCR is strongly expressed throughout the entire dT1 region (Fig. 3A upper left, arrowhead). At the same time, in another orthopteran Schistocerca (grasshoppers), the signal is observed in discrete regions of dT1 (Fig. 3A upper right, arrowhead). The remaining insect lineages examined here (Periplaneta, Tenodera, and Oncopeltus) all exhibit strong expression of SCR throughout the entire dT1 region similar to the pattern observed in Acheta. This observation is consistent with the fact that Scrhas recently been found to repress wing formation on the prothoracic segment in hemimeatabolus species as well (Chesebro et al., 2009).

\section{SCR patterning in the T1 leg is very dynamic and character- ized by a frequent gain and loss of expression domains}

Insect legs exhibit a range of morphological diversity encompassing small-scale (bristle pattern, coloration) and large-scale (overall size and shape) differences. Despite such vast diversity, all 
insect legs share a common modular organization and are composed of the same five basic segments: coxa, trochanter, femur, tibia, and tarsus, followed by claws. One of the distinguishable features of prothoracic legs in some species is the presence of a row of bristles (sex combs) in discrete leg segments. Functional studies in Drosophila, Tribolium, and Oncopeltushave all indicated that $\mathrm{Scr}$ directs the formation of this T1 leg-specific structure (Beeman et al., 1989; Hughes and Kaufman, 2000; Pattatucci et al., 1991). However, there are many more modifications of prothoracic legs that are distributed across insect groups. The best known example of such extreme modification is observed in praying mantids, where $\mathrm{T} 1$ legs are transformed into large raptorial-like appendages that use rows of unique spurs to capture and hold prey. What remains unclear is whether and to what degree Scrmay be playing a role in the development of these highly modified structures. At the same time, other insect lineages have prothoracic legs that do not bear any unique features and are morphologically very similar to T2 legs. This leads to another important question: is SCR still expressed in the T1 legs of these species? Note that all of the insect species analyzed in this report undergo a hemimetabolous mode of development, thereby allowing a direct association between SCR expression and distinct T1 leg morphology of first nymphs.

The T1 legs of the firebrat are morphologically similar to their T2 and T3 counterparts, bearing no defining structural features such as combs or spurs. Consistent with this phenotype, neither SCR protein (Fig. 1F) nor its mRNA (Rogers et al., 1997) is expressed in the T1 legs at any time during Thermobia development.

Despite the fact that they belong to the same group, the available expression data in three orthopteran species (Acheta, Gryllus and Schistocerca) reveal the presence of divergent SCR leg patterning among these lineages. At $\sim 35 \%$ development, the T1 leg of Acheta accumulates both Scr mRNA (Rogers et al., 1997) and protein (Fig. 3B, arrowhead) in a small patch of cells in the anterior region that corresponds to the future tibial-tarsal joint. In Gryllus, another cricket species, ScrmRNA has a larger domain encompassing the trochanter, femur,

Fig. 4. Generalized summary of SCR protein expression patterns of the insect species examined in this study. The large rectangle on the left represents the midventral region of the head and first thoracic segments separated by dashed lines. The smaller rectangles on the right correspond to the associated appendages of these segments. Blue domains indicate early SCR expression and orange domains represent signal that appears only at later stages of development. Striped blue and orange domains indicate expression that occurs at both early and late developmental stages. A red asterisk denotes regions in which SCR protein accumulation differs from previously reported mRNA patterns (Rogers et. al 1997). Abbreviations: $M n$, mandible; $M x$, maxillae; Lb, labium, dT1 dorsal T1 region. tiba and tarsus (Zhang et al., 2005). However, in both instances there are no obvious unique $\mathrm{T} 1$ leg features that can be associated with these observed expression patterns. This suggests that the expansion of SCR into a novel domain (T1 leg) preceded the apparent gain of function. In contrast, grasshoppers never accumulate SCR protein in the T1 leg at any time during development (Fig. 3C). In orthopterans therefore, SCR protein patterns in the prothoracic leg are labile and cannot be linked to any specific T1 leg trait.

In the two dictyopteran species (cockroach and praying man-

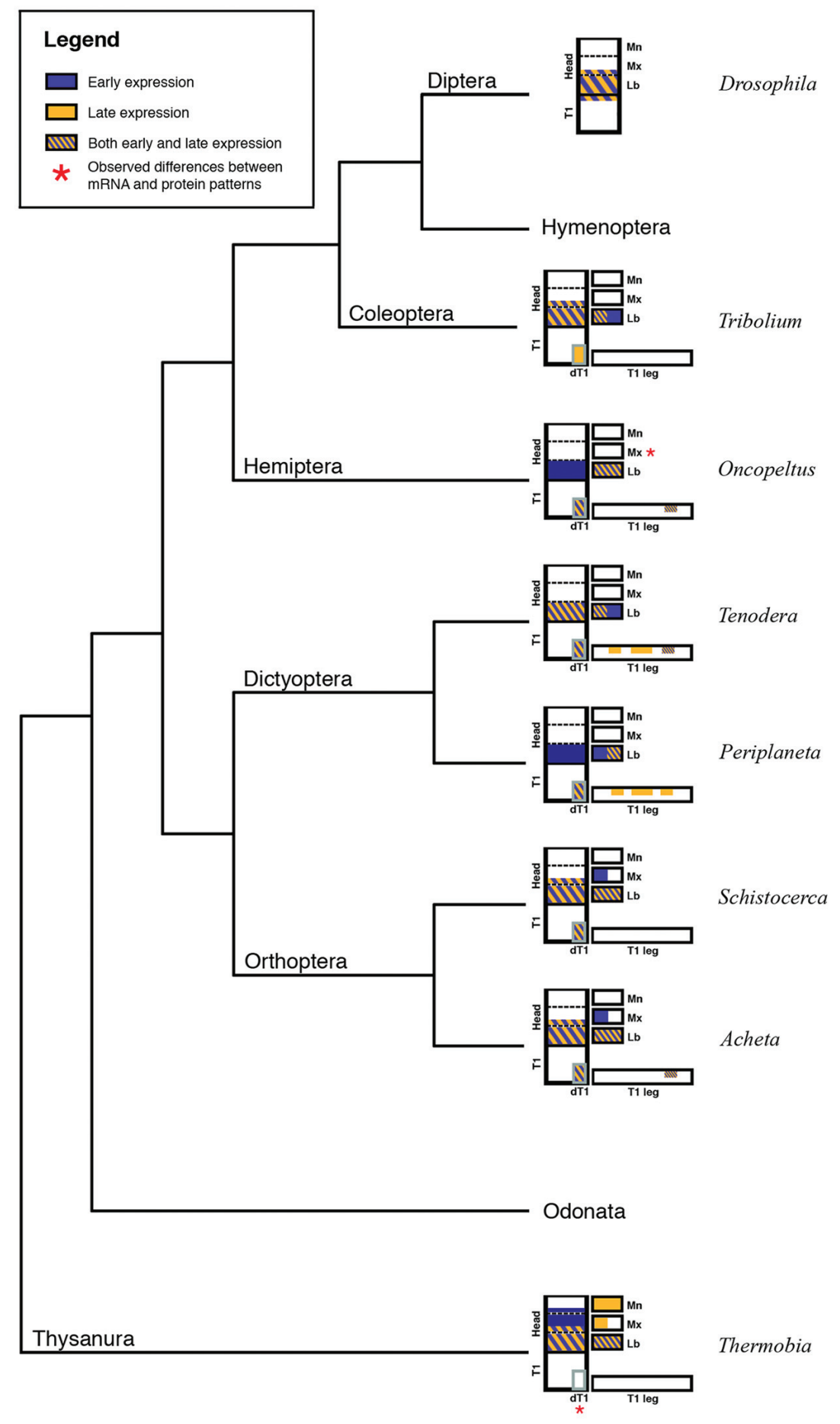


tis), SCR accumulates in multiple regions along the T1 legs but only at later stages of development, around the time when leg segmentation becomes evident. As shown in Fig. 3D, no SCR protein can be detected in the T1 legs of cockroach embryos at earlier embryonic stages ( $30 \%)$. At $\sim 45 \%$ development, strong signal is detected in most of the tibia and in the distal half of the femur (Fig. 3D, right). In addition, a more moderate signal is also present in a smaller cluster of cells in the proximal region of the coxa. While the T1 legs of the cockroach exhibit spur-like outgrowths along the posterior border of these developing appendages, they are not unique structures as both T2 and T3 legs have a similar morphology. SCR accumulation in the T1 legs of Periplaneta therefore likely represents another variation of expression with no apparent phenotype, similar to the situation observed in orthopterans.

Compared to cockroaches, SCR expression in mantids ( Tenodera) starts at earlier developmental stages in a small patch of cells restricted to the anterior margin of the tibia (Fig. 3E). At $\sim 45 \%$ development, SCR patterning in the T1 leg changes and becomes confined to the opposite (posterior) edge encompassing four discrete domains: the coxa, the anterior and posterior femur separated by a narrow gap, and the tibia (Fig. 3E bottom left). As depicted in Fig. 3E (right), the prothoracic legs of mantids are highly modified appendages characterized by the presence of unique rows of spurs on the distal femoral and tibial segments. Hence, the later SCR expression patterns in these segments can be directly associated with this T1-leg specific morphology. At the same time, the SCR signal in the coxa and proximal femur do not correspond to any particular phenotype as these regions are devoid of any defining characteristic. Thus, in Tenoderawe have a situation where the distal half of SCR expression is associated with the development of the grasping apparatus on $\mathrm{T} 1$, while the proximal half has no apparent function.

Finally, both mRNA expression and functional analyses in Oncopeltus (Hughes and Kaufman, 2000; Rogers et al., 1997; Rogers et al., 2002) have shown that Scrgoverns the formation of the sex combs on the distal tibia of the T1 leg (Fig. 3F inset, top). Consistent with these data, SCR protein accumulates in the same region (distal tibia) as the previously reported mRNA expression patterns (Fig. 3F). Studies in Drosophila and Tribolium have shown that the ability of SCR to direct the formation of T1 leg combs may be a shared feature in holometabolous species, as hypomorphic alleles in these lineages result in the abolition of this structure (Beeman et al., 1989; Pattatucci et al., 1991). Overall, these data suggest that SCR may also function in directing the formation of T1-specific structures in other insects, such as the grasping apparatus present in mantids.

\section{Discussion}

Studies utilizing cross-reacting antibodies can provide valuable insight into the evolution of expression patterns, and to a certain degree, gene function(s) in a broad range of taxa. Our global analysis of SCR protein accumulation in hemimetabolous species shows that the expression patterns of this gene are highly dynamic and species-specific. As illustrated in Fig. 4, there are five trends with regard to the evolution of the expression and putative function of this gene in insects. First, the domain of SCR is confined to the head with no expression in the dT1 or protho- racic regions in the basal insect lineage Thermobia. Second, in this species, the most anterior border of expression is in the mandibular segment. Rogers et al. (1997) originally reported that the anterior boundary of ScrmRNA accumulation is in the posterior maxillary region. Note, however, that the earliest embryo used in that analysis is at a mid-developmental stage ( 40\%), similar to the one shown in our Fig. 1C. Consistent with the mRNA data, the mandibles also lack SCR protein accumulation at this stage. However, in the present analysis we were able to obtain SCR expression patterns at both earlier and later stages of development ( $\sim 30 \%$ and $\sim 75 \%$, respectively) that show novel accumulation in the mandibles (Fig. $1 \mathrm{~A}, \mathrm{E}$ ). Thermobia therefore displays the most anterior domain of SCR expression reported to date. Third, with the exception of the firebrats, all species analyzed in this report have expression in the $\mathrm{dT} 1$ region. Several studies in more derived insect species have indicated that Screxpression in $\mathrm{dT} 1$ is a conserved feature and functions in suppressing wing development on this segment (Beeman et al., 1989; Carroll et al., 1995; Chesebro et al., 2009; Rogers et al., 1997). Note however, that the firebrat represents a primitively wingless species. This observation led to the original proposition that the expression of ScrmRNA in the dT1 region likely predates the origin of wings (Rogers et al., 1997). Our data supports this view and further suggests that the presence of mRNA in the firebrat dT1 reveals the beginning of an expansion of Scrinto the prothorax. Fourth, while ScrmRNA transcript can be detected in the dT1 of firebrats and the maxillary segment of milkweed bugs (Rogers etal., 1997), no eventual protein accumulates in these two regions (Fig. 4, asterisks). The observed discrepancy between mRNA and protein accumulation suggests the presence of a post-transcriptional regulatory mechanism, similar to the one previously reported in crustaceans (Abzhanov and Kaufman, 1999). Finally, SCR expression in the prothoracic legs of different insect species is highly labile and can be gained or lost easily. In addition, it is only in more late-branching lineages that domains of expression can be directly associated with $\mathrm{T} 1$-specific morphologies suggesting that the acquisition of SCR in the prothoracic leg may have preceded any apparent gain of function.

The availability of both protein and mRNA expression patterns provides for a much more detailed understanding of the functions of $S c r$ in insects. For example, the original report of mRNA patterning in Oncopeltus showed that Scris primarily localized in the labial segment. The signal is also confined to a few cells in the maxillary region and is never observed in the mandibles (Rogers et al., 1997). Subsequent embryonic RNAi analysis confirmed that the primary influence of Scris in the labial segment, indicated by the transformation of this appendage toward a leg-like identity (Hughes and Kaufman, 2000). However, phenotypic changes were also observed in the mandibular and maxillary stylets which were transformed into a mass of undifferentiated tissue. This result was inconsistent with the observed mRNA pattern leading to the suggestion that non-local indirect effects may be responsible for generating such phenotypes (Hughes and Kaufman, 2000). The present study provides an independent corroboration of this view by showing that indeed no SCR protein is present in these segments at any time during development. Hence, the function of Scrin the head region of Oncopeltusis restricted to the labial segment only, and should have no effect on the development of the more anterior mouthparts. Similarly, previous studies 
in Drosophila and Triboliumshowed that Scr Cxmutations do not affect the maxillary appendages (Beeman et al., 1989; Curtis et al., 2001; Pattatucci et al., 1991; Shippy et al., 2006; Wakimoto and Kaufman, 1981), suggesting that restriction of its function to the labium preceded the divergence between hemimetabolous and holometabolous insect lineages. As illustrated by Fig. 4, among hemimetabolous species, the orthopterans have SCR expression in both the maxillary and labial appendages and represent an ideal choice for future extension of functional studies. Such studies will be able to delineate Scrfunction in both of these segments, thus providing a more complete understanding of this gene's role in the evolution of insect mouthparts.

The current study also reveals a trend toward the continuous posteriorization of the anterior border of SCR expression in the insect head consistent with the divergence of insect lineages from early to late-branching. While the basal insect species Thermobia shows clear signal in the mandibles at early and late developmental stages, the most anterior expression of SCR is in the maxillary segment in the orthopterans (cricket and grasshopper). An identical mRNA pattern has been reported in another cricket species (Gryllus bimaculatas), suggesting that the maxillary border or $\mathrm{Scr}$ expression may be a conserved feature within the orthopterans (Zhang et al., 2005). Finally, dictyopterans and hemipterans (cockroach, mantis, and milkweed bug) all have an anterior border of SCR expression in the labial segment. Due to the fact that Thermobia represents an early-branching insect group, it is tempting to speculate that SCR patterning in this lineage represents the ancestral anterior border of expression in the insect head. Alternatively, it is possible that Thermobia acquired a derived character state that is species-specific. In order to distinguish between these two alternatives it is necessary to extend SCR expression analyses to encompass other more early-branching lineages (such as Odonata) in order to truly delineate what is the ancestral patterning in the head region. It is equally important to note that studies of SCR protein accumulation in the highly derived holometabolous insects Drosophila and Tribolium show that the anterior expression boundary reverts back to the posterior portion of the maxillary segment (Carroll et al., 1988; Curtis et al., 2001; Riley et al., 1987; Shippy et al., 2006). This result suggests that while SCR protein expression was lost in the maxillary segment of late-branching hemimetabolous lineages, it was subsequently regained prior to the divergence of the Holometabola.

Classical studies in Drosophila and Tribolium have indicated that one of the primary functions of Scris to provide identity to the prothoracic (T1) segment and that this role is likely conserved in all insects (Beeman et al., 1989; Curtis et al., 2001; Pattatucci et al., 1991; Shippy et al., 2006; Wakimoto and Kaufman, 1981). Furthermore, recent insight from Oncopeltus shows that in hemimetabolous species, the role of Scrin directing T1 morphology is restricted to post-embryonic development (Chesebro et al., 2009). This finding highlights the importance of determining the role(s) of Scrin the prothorax of other insect lineages such as orthopterans and dictyopterans. In addition, many hemimetabolous species display extreme morphological modifications of their T1 segments that are established during post-embryogenesis. Except for Oncopeltus, virtually no data is currently available regarding the potential role Scrmay play in the divergence of the prothorax during this stage of development. Addressing these questions will be necessary for determining the full extent of Scrinvolvement in the evolution of T1 morphology in hemimetabolous insect species.

\section{Materials and Methods}

The laboratory cultures of firebrats (Thermobia domestica), crickets (Acheta domesticus) and milkweed bugs (Oncopeltus fasciatus) were reared under conditions previously described in Rogers et al.,1997) and Peterson et al.,1999). The egg cases of the cockroach (Periplaneta americana) and the praying mantis ( Tenodera aridifolia) were purchased from Carolina Biological Supply Company and were used to establish laboratory cultures. The grasshopper (Schistocerca americana) embryos were gifts from Markus Friedrich (Wayne State University).

Dissection and fixation of embryos was performed as previously described in Mahfooz et al. (2004). Expression was detected by using a rat polyclonal antibody generated against a C-terminal fragment of Drosophila SCR and kindly donated by D.J. Andrew and M.P. Scott (unpublished). This antibody was subsequently described and was found to cross-react to SCR in crustaceans (Abzhanov and Kaufman, 1999) and Tribolium (Curtis et al., 2001). In this study, we confirmed that the protein patterns produced by this antibody closely mirror signal observed in parallel in situhybridization experiments in Periplaneta and Oncopeltus (Supp. Fig. 1). The staining was performed as described in Mahfooz et al. (2004). The antibody was detected by using a secondary anti-rat antibody that was conjugated to FITC, horseradish peroxidase, or alkaline phosphatase (The Jackson Laboratory). Detailed protocols on maintaining insect cultures, collection of embryos and antibody staining are available upon request.

\section{Acknowlegements}

We would like to thank Debbie Andrew for generously providing the SCR antibody used in this study and Markus Friedrich for the donation of grasshopper embryos. We also thank two anonymous reviewers whose comments greatly improved the manuscript. This work was supported by $\mathrm{NIH}$ grant GM071927 to A.P. and a Wayne State University graduate student enhancement fund to $S$. $H$.

\section{References}

ABZHANOV, A. and KAUFMAN, T.C. (1999). Novel regulation of the homeotic gene Scr associated with a crustacean leg-to-maxilliped appendage transformation. Development 126: 1121-1128.

ABZHANOV, A., POPADIC, A. and KAUFMAN, T.C. (1999). Chelicerate Hox genes and the homology of arthropod segments. Evol Dev1: 77-89.

ANGELINI, D.R. and KAUFMAN, T.C. (2005). Functional analyses in the milkweed bug Oncopeltus fasciatus (Hemiptera) support a role for Wnt signaling in body segmentation but not appendage development. Dev Bio/283: 409-423.

AVEROF, M. and PATEL, N.H. (1997). Crustacean appendage evolution associated with changes in Hox gene expression. Nature 388: 682-686.

BEEMAN, R.W., STUART, J.J., BROWN, S.J. and DENELL, R.E. (1993). Structure and function of the homeotic gene complex (HOM-C) in the beetle, Tribolium castaneum. Bioessays 15: 439-444.

BEEMAN, R.W., STUART, J.J., HAAS, M.S. and DENELL, R.E. (1989). Genetic analysis of the homeotic gene complex (HOM-C) in the beetle Tribolium castaneum. Dev Biol133: 196-209.

CARROLL, S.B., DINARDO, S., O'FARRELL, P.H., WHITE, R.A. and SCOTT, M.P (1988). Temporal and spatial relationships between segmentation and homeotic gene expression in Drosophila embryos: distributions of the fushi tarazu, engrailed, Sex combs reduced, Antennapedia, and Ultrabithorax proteins. Genes Dev 2: 350-360

CARROLL, S.B., GRENIER, J.K. and WEATHERBEE, S.D. (2001). From DNA to diversity: Molecular Genetics and the evolution of animal design. Blackwell Science Inc., Malden, Massachusetts. 
CARROLL, S.B., WEATHERBEE, S.D. and LANGELAND, J.A. (1995). Homeotic genes and the regulation and evolution of insect wing number. Nature 375: 5861.

CASTELLI-GAIR, J. and AKAM, M. (1995). How the Hox gene Ultrabithorax specifies two different segments: the significance of spatial and temporal regulation within metameres. Development 121: 2973-2982.

CHESEBRO, J., HRYCAJ, S., MAHFOOZ, N. and POPADIC, A. (2009). Diverging functions of Scr between embryonic and post-embryonic development in a hemimetabolous insect, Oncopeltus fasciatus. Dev Bio/329: 142-151.

CURTIS, C.D., BRISSON, J.A., DECAMILLIS, M.A., SHIPPY, T.D., BROWN, S.J. and DENELL, R.E. (2001). Molecular characterization of Cephalothorax, the Tribolium ortholog of Sex combs reduced. Genesis 30: 12-20.

DAMEN, W.G., HAUSDORF, M., SEYFARTH, E.A. and TAUTZ, D. (1998). A conserved mode of head segmentation in arthropods revealed by the expression pattern of Hox genes in a spider. ProcNat/Acad SciUSA95: 10665-10670.

HUGHES, C.L. and KAUFMAN, T.C. (2000). RNAi analysis of Deformed, proboscipedia and Sex combs reduced in the milkweed bug Oncopeltus fasciatus: novel roles for Hox genes in the hemipteran head. Development 127: 3683-3694.

HUGHES, C.L. and KAUFMAN, T.C. (2002). Hox genes and the evolution of the arthropod body plan. Evol Dev 4: 459-499.

KELSH, R., WEINZIERL, R.O., WHITE, R.A. and AKAM, M. (1994). Homeotic gene expression in the locust Schistocerca: an antibody that detects conserved epitopes in Ultrabithorax and abdominal-A proteins. Dev Genet 15: 19-31.

MAHAFFEY, J.W. and KAUFMAN, T.C. (1987). Distribution of the Sex combs reduced gene products in Drosophila melanogaster. Genetics 117: 51-60.

MAHFOOZ, N., TURCHYN, N., MIHAJLOVIC, M., HRYCAJ, S. and POPADIC, A. (2007). Ubx regulates differential enlargement and diversification of insect hind legs. PLOS ONE2: e866.

MAHFOOZ, N.S., LI, H. and POPADIC, A. (2004). Differential expression patterns of the hox gene are associated with differential growth of insect hind legs. Proc Natl Acad Sci USA 101: 4877-4882.

PATTATUCCI, A.M. and KAUFMAN, T.C. (1991). The homeotic gene Sex combs reduced of Drosophila melanogasteris differentially regulated in the embryonic and imaginal stages of development. Genetics 129: 443-461.

PATTATUCCI, A.M., OTTESON, D.C. and KAUFMAN, T.C. (1991). A functional and structural analysis of the Sex combs reduced locus of Drosophila melano- gaster. Genetics 129: 423-441.

PETERSON, M.D., ROGERS, B.T., POPADIC, A. and KAUFMAN, T.C. (1999). The embryonic expression pattern of labial, posterior homeotic complex genes and the teashirt homologue in an apterygote insect. Dev Genes Evo/209: 77-90.

RILEY, P.D., CARROLL, S.B. and SCOTT, M.P. (1987). The expression and regulation of Sex combs reduced protein in Drosophila embryos. Genes Dev1: 716-730.

ROGERS, B.T., PETERSON, M.D. and KAUFMAN, T.C. (1997). Evolution of the insect body plan as revealed by the Sex combs reduced expression pattern. Development 124: 149-157.

ROGERS, B.T., PETERSON, M.D. and KAUFMAN, T.C. (2002). The development and evolution of insect mouthparts as revealed by the expression patterns of gnathocephalic genes. Evol Dev 4: 96-110.

SHIPPY, T.D., ROGERS, C.D., BEEMAN, R.W., BROWN, S.J. and DENELL, R.E. (2006). The Tribolium castaneum ortholog of Sex combs reduced controls dorsal ridge development. Genetics 174: 297-307.

STERN, D.L. (1998). A role of Ultrabithorax in morphological differences between Drosophila species. Nature 396: 463-466.

STRUHL, G. (1982). Genes controlling segmental specification in the Drosophila thorax. Proc Natl Acad Sci USA 79: 7380-7384.

TELFORD, M.J. and THOMAS, R.H. (1998). Expression of homeobox genes shows chelicerate arthropods retain their deutocerebral segment. Proc Natl Acad Sci USA 95: 10671-10675.

TOMOYASU, Y., WHEELER, S.R. and DENELL, R.E. (2005). Ultrabithorax is required for membranous wing identity in the beetle Tribolium castaneum. Nature 433: 643-647.

WAKIMOTO, B.T. and KAUFMAN, T.C. (1981). Analysis of larval segmentation in lethal genotypes associated with the antennapedia gene complex in Drosophila melanogaster. Dev Biol81: 51-64

ZHANG, H., SHINMYO, Y., MITO, T., MIYAWAKI, K., SARASHINA, I., OHUCHI, H. and NOJI, S. (2005). Expression patterns of the homeotic genes Scr, Antp, Ubx, and abd-A during embryogenesis of the cricket Gryllus bimaculatus. Gene Expr Patterns 5: 491-502.

ZHENG, Z., KHOO, A., FAMBROUGH, D., JR., GARZA, L. and BOOKER, R. (1999). Homeotic gene expression in the wild-type and a homeotic mutant of the moth Manduca sexta. Dev Genes Evo/209: 460-472.

\section{Further Related Reading, published previously in the Int. J. Dev. Biol.}

See Special Issue Pattern Formation edited by Michael K. Richardson and Cheng-Ming Chuong at: http://www.ijdb.ehu.es/web/contents.php?vol=53\&issue=5-6

\section{Function and specificity of Hox genes}

David Foronda, Luis F. de Navas, Daniel L. Garaulet and Ernesto Sánchez-Herrero Int. J. Dev. Biol. (2009) 53: 1409-1419

\section{Evolution of the Hox/ParaHox gene clusters}

David E K Ferrier and Carolina Minguillón

Int. J. Dev. Biol. (2003) 47: 605-611

The origin and evolution of appendages.

Alessandro Minelli

Int. J. Dev. Biol. (2003) 47: 573-581

Understanding the genetic basis of morphological evolution: the role of homeotic genes in the diversification of the arthropod bauplan A Popadic, A Abzhanov, D Rusch and T C Kaufman Int. J. Dev. Biol. (1998) 42: 453-461

Implications of the spatial and temporal regulation of Hox genes on development and evolution

$\mathrm{J}$ Castelli-Gair

Int. J. Dev. Biol. (1998) 42: 437-444
5 yr ISI Impact Factor $(2008)=3.271$

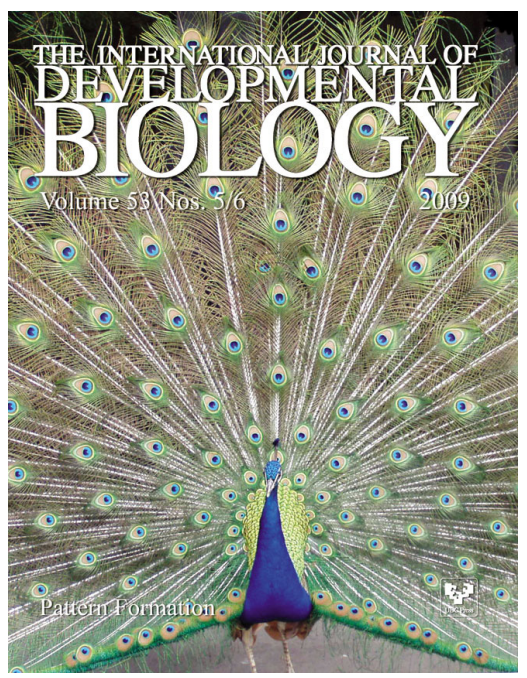

